\title{
Establishment of the chloroplast genetic system in rice during early leaf development and at low temperatures
}

\section{Kensuke Kusumi* and Koh lba}

Department of Biology, Faculty of Sciences, Kyushu University, Fukuoka, Japan

\section{Edited by:}

Thomas Pfannschmidt, University Joseph Fourier Grenoble, France

\section{Reviewed by:}

Wataru Sakamoto, Okayama

University, Japan

Ben Field, Centre National de la

Recherche Scientifique, France

\section{${ }^{*}$ Correspondence:}

Kensuke Kusumi, Department of

Biology, Faculty of Sciences, Kyushu

University, Fukuoka 812-8581, Japan

e-mail: kusumi.k.239@m.kyushu-u.ac.jp

\begin{abstract}
Chloroplasts are the central nodes of the metabolic network in leaf cells of higher plants, and the conversion of proplastids into chloroplasts is tightly coupled to leaf development. During early leaf development, the structure and function of the chloroplasts differ greatly from those in a mature leaf, suggesting the existence of a stage-specific mechanism regulating chloroplast development during this period. Here, we discuss the identification of the genes affected in low temperature-conditional mutants of rice (Oryza sativa). These genes encode factors involved in chloroplast rRNA regulation (NUS1), and nucleotide metabolism in mitochondria, chloroplasts, and cytosol $\left(V_{2}, V_{3}, S T 1\right)$. These genes are all preferentially expressed in the early leaf developmental stage P4, and depleting them causes altered chloroplast transcription and translation, and ultimately leaf chlorosis. Therefore, it is suggested that regulation of cellular nucleotide pools and nucleotide metabolism is indispensable for chloroplast development under low temperatures at this stage. This review summarizes the current understanding of these factors and discusses their roles in chloroplast biogenesis.
\end{abstract}

Keywords: chloroplast transcription, translation, Oryza sativa, low temperature, nucleotide metabolism

\section{INTRODUCTION}

Low temperature is a major abiotic constraint to plant growth. In rice, two stages of development are known to be the most sensitive to low temperatures the young seedling stage and the booting stage (Kaneda and Beachell, 1974; Cruz et al., 2013). At the booting stage, pollen sterility caused by low temperatures decreases the final grain yield. At the seedling stage, low temperatures reduce germination and delay leaf emergence and greening. Leaf chlorosis and yellowing are common symptoms when a low temperature prevails during this stage (Cruz et al., 2013), which suggests the low temperature arrests chloroplast development and functioning.

The effect of a low-temperature environment on chloroplast functions has been studied extensively (Berry and Björkman, 1980; Hasanuzzaman etal., 2013). A low temperature causes swelling of the thylakoid lamellae, vesiculation of the thylakoid, and ultimately breakdown of the entire chloroplast. A low temperature also inhibits electron transport and the carbon assimilation apparatus such as the Calvin cycle, ATP synthase, and ribulose 1,5-bisphosphate carboxylase/oxygenase (RuBisCO; Demmig-Adams and Adams, 1992; Asada, 1999; Yamori etal., 2011; Hasanuzzaman etal., 2013). However, these physiological symptoms have been mainly investigated in mature leaves containing functionally established chloroplasts. The molecular mechanisms underlying early chloroplast development under low temperatures have not yet been extensively studied.

virescent is a chlorotic mutant of higher plants causing young leaves to have a reduced chlorophyll content, but the chlorophyll levels recover as they grow (Archer and Bonnett, 1987). In contrast with other chlorotic mutants showing lethality such as albino, chlorina, and xantha, the virescent mutants are not terminal, and can reach maturity and produce seeds. Certain classes of the virescent mutations that have been reported are low-temperature conditional. They develop chlorotic leaves under low temperatures, but not under higher temperatures, suggesting a temporal aberration in a factor governing chloroplast development under low-temperature conditions. During the past decade, numerous genes responsible for virescent mutations have been identified in rice, and they have been shown to be involved in the chloroplast genetic system, including transcription, translation, and nucleotide metabolism. Because many of these genes are expressed temporally during early leaf development, they are probably involved in the establishment of the plastid genetic system at this phase under low temperatures. Here, we introduce four factors involved in chloroplast biogenesis under low-temperature conditions (NUS1, GKpm, RNRS1, and RNRL1) that have been identified through genetic and functional analysis of virescent mutants of rice $\left(v_{1}, v_{2}, v_{3}\right.$, and $s t 1)$.

\section{TEMPERATURE-SENSITIVE PHASE OF virescent MUTANTS DURING EARLY LEAF DEVELOPMENT}

virescent $-1,-2$, and $-3\left(v_{1}, v_{2}, v_{3}\right)$ were the first virescent mutants reported in rice and the mutations have been used as classical genetic markers (Omura et al., 1977). They develop chlorotic leaves at a restrictive low temperature $\left(20^{\circ} \mathrm{C}\right)$ but nearly normal green leaves at a permissive higher temperature $\left(30^{\circ} \mathrm{C}\right.$; Figure 1A). They are often hard to distinguish from each other, showing similar phenotypes. An important characteristic of these virescent mutants is that the leaf phenotype is not influenced by growth temperature after its emergence (Iba etal., 1991). This indicates that the leaf phenotype is irreversibly determined by 
the environmental temperature at a certain developmental stage before emergence. Furthermore, this phenotype can be useful for determining the temperature-sensitive period (TSP), by shifting the temperature from restrictive to permissive during leaf development, or vice versa. This technique was originally performed with Drosophila (Suzuki, 1970), in which the TSP for conditional mutants was limited to particular stages of development. Temperature-shift experiments showed that the TSPs of all $v_{1}, v_{2}$, and $v_{3}$ mutants was at stage P4 of leaf development (Iba et al., 1991).

Rice has the striking feature of leaf primordia production (plastochron) that is synchronized with leaf emergence (phyllochron) in shoot development (Nemoto and Yamazaki, 1993; Itoh et al., 2005). This regularity of leaf development enables a series of successive stages to be defined, starting with P0 (leaf founder cells), through P1 (youngest primordium), P2, P3, P4, and P5, to P6 (a fully expanded leaf; Figure 1B). Anatomical studies have shown that in rice the $\mathrm{P} 4$ stage is characterized by rapid leaf blade elongation (Itoh et al., 2005). Leaves at the P4 stage have an initial length of 3-5 mm and reach a final size of about $8-10 \mathrm{~cm}$. Chlorophyll concentration per unit of fresh weight is negligible in the early P4 stage, and increases to about $40 \%$ in a mature leaf (Kusumi et al., 2010a). Electron microscopic observations have indicated that chloroplasts in the leaves at the early $\mathrm{P} 4$ stage have a small spherical shape (below $1 \mu \mathrm{m}$ ) and poor internal thylakoid structures. After the mid-P4 stage, thylakoid extension and grana formation in chloroplasts has been observed within the mid-portion of the leaf (Kusumi et al., 2010b).

The process of chloroplast development is divided roughly into three steps: (i) plastid division and DNA replication; (ii) establishment of the plastid genetic system; and (iii) activation of the photosynthetic apparatus (Jarvis and Lopez-Juez, 2013; Yagi and Shiina, 2014). These stepwise processes are partially achieved by two plastidial RNA polymerases; a nucleus-encoded phage-type RNA polymerase (NEP), and a plastid-encoded bacterial-type RNA polymerase (PEP; Hajdukiewicz et al., 1997; Yagi and Shiina, 2014). Plastid genes involved in the second step are known to be mainly transcribed by NEP, and those involved in the third step are transcribed by PEP (Yagi and Shiina, 2014). Analyses for chloroplast transcript accumulation revealed that the first step of chloroplast differentiation is likely to start in the leaves at the P0-P3 stages, and will largely finish during the early P4 stage (Figure 1C; Kusumi et al., 2010b). The second step occurs significantly in the leaves at mid-P4, and the decline of the second step and onset of the third step take place during late P4 (Kusumi et al., $2010 \mathrm{~b}$ ). The accumulation of tRNA ${ }^{\mathrm{Glu}}$, a bifunctional molecule mediating the early steps of chlorophyll synthesis, and the switching of transcription from NEP to PEP (Hanaoka et al., 2005), showed two peaks (late-P4 and P5). The first activation of tRNA Glu can be related to the NEP-PEP transition. Therefore, the TSP of $v_{1}$, $v_{2}$, and $v_{3}$ mutants at the $\mathrm{P} 4$ stage suggests that they may be related to the establishment of the chloroplast genetic system, which is the major process occurring at this stage.

\section{REGULATION OF CELLULAR NUCLEOTIDE POOLS INVOLVED IN THE CHLOROPLAST DEVELOPMENT}

Virescent-2 $\left(V_{2}\right)$ was the first gene isolated from virescent mutants of rice (Sugimoto et al., 2007). Functional analyses showed that $V_{2}$ encoded guanylate kinase (GK), a key enzyme in guanine nucleotide biosynthesis that catalyzes the conversion of GMP to
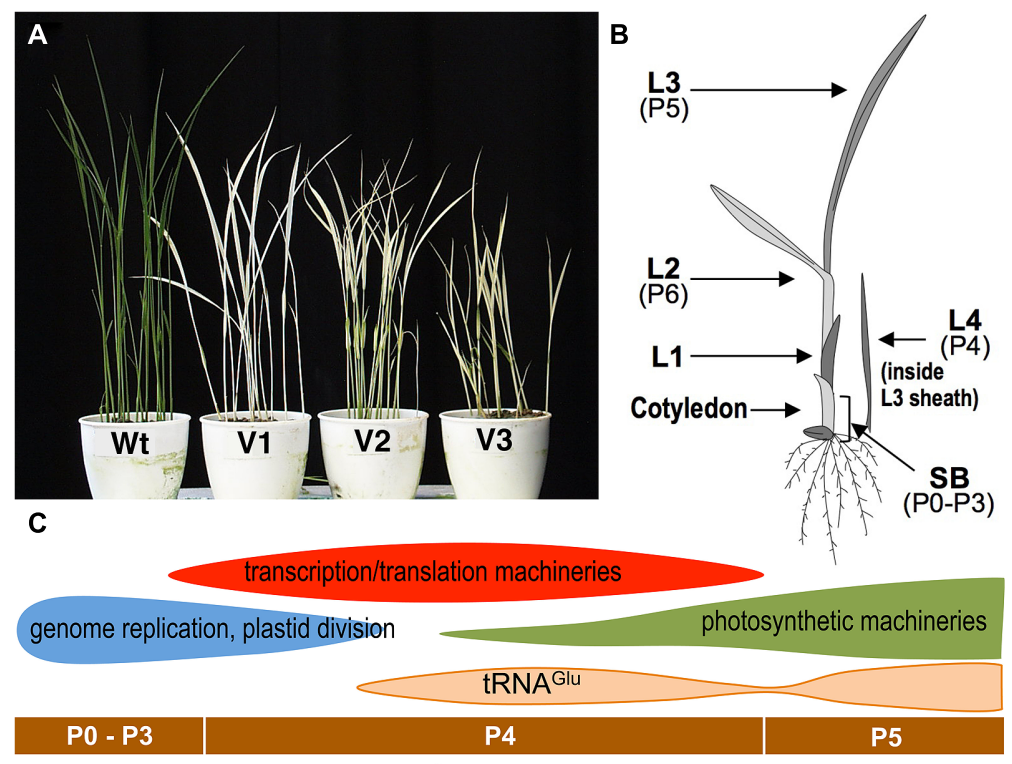

Leaf developmental stage

FIGURE 1 I (A) Phenotypes of the wild-type (Wt) and virescent mutants ( $v_{1}$, $v_{2}$, and $\left.v_{3}\right)$ grown at a restrictive temperature $\left(20^{\circ} \mathrm{C}\right)$. (B) Schematic of a rice seedling with a fully expanded third leaf. L1, L2, L3, and L4 indicate the first, second, third, and fourth leaf, respectively. Developmental stages (P0-P6) are also indicated. shoot base, SB corresponds to a $5 \mathrm{~mm}$ section from the bottom of the shoot and contains pre-emerged leaves at P0-P3 stages. (C) Plastid gene expression patterns during leaf development (Kusumi etal., 2010a). Horizontal bars indicate the leaf developmental stages. 


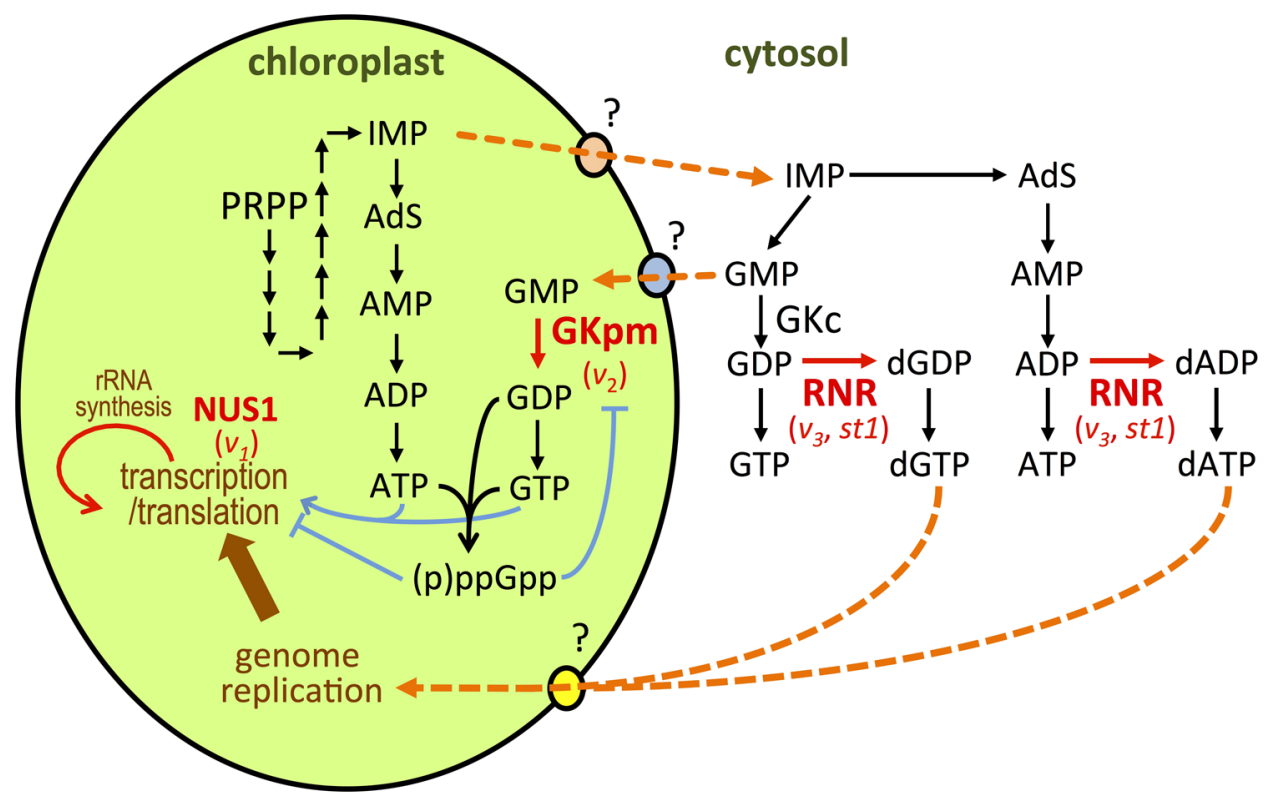

FIGURE 2 | Representation of de novo purine nucleotide synthesis in chloroplasts and cytosol (Sugimoto et al., 2007; Yoo et al., 2009; Kusumi et al., 2011; Nomura et al., 2014). Proteins whose function has been linked to chloroplast development at cold temperature are depicted in red. The names of the mutation for each protein are indicated below. Orange dotted arrows indicate hypothetical routes for membrane transport or enzyme conversions. PRPP, 5-phosphoribosyl 1-pyrophosphate; IMP, 5'-monophosphate; AdS, adenylosuccinat.
GDP (Figure 2). In bacterial and animal species, GK is localized in the cytoplasm and participates in maintenance of the guanine nucleotide pools. Plants possess two types of GK; cytosolic GK (GKc) and plastid/mitochondrial GK (GKpm; Sugimoto etal., 2007). Analysis of RNAi knockdown plants showed that GKc is essential for the growth and development of plants, but not for chloroplast development (Sugimoto et al., 2007). $V_{2}$ is a single-copy gene encoding the GKpm protein. $V_{2}$-encoded GKpm predominantly accumulates in developing leaves at the P0-P4 stages (Sugimoto et al., 2007), which is consistent with a temperature-shift experiment in which the $V_{2}$ gene product was shown to be necessary at the P4 stage. A chloroplast possesses its own nucleoside diphosphate kinase that catalyzes subsequent GDP to GTP conversion (Figure 2; Sugimoto et al., 2007; Kihara et al., 2011; Nomura et al., 2014). Therefore, GKpm can limit the GDP/GTP pool in the chloroplast. Reduction of GKpm activity will cause a shortage of the GTP necessary for the assembly and function of the plastid translation machinery. In the $v_{2}$ mutant, Val162 has been substituted with Ile, which caused a 20 -fold reduction in specific GMP activity (Sugimoto et al., 2007), and severely suppresses chloroplast translation (Sugimoto et al., 2004). Similarly, bacterial GTPases have important roles in ribosome biogenesis and protein translation (Verstraeten et al., 2011). In Arabidopsis and tobacco, plastidial GTPases have been reported to be involved in chloroplast rRNA processing and ribosome biogenesis in higher plants (Jeon et al., 2014). It has also been reported that an Arabidopsis mutant deficient in GTP-dependent chloroplast elongation factor G developed pale cotyledons and greenish true leaves, as observed in the GKpmdeficient Arabidopsis (Albrecht et al., 2006; Sugimoto et al., 2007).
This phenotypic similarity suggests the involvement of GKpm in the regulation of plastid translation, via limitation of the GTP pool.

Additionally, it was recently reported that GKpm is a target of regulation by guanosine $3^{\prime}, 5^{\prime}$-bisdiphosphate (ppGpp) in chloroplasts of rice, as well as those of peas and Arabidopsis (Nomura etal., 2014). In bacteria, ppGpp is a key regulatory molecule that controls the stringency of responses through direct interaction with protein factors involved in gene expression such as RNA polymerase, translation factors, and DNA primase (Potrykus and Cashel, 2008; Tozawa and Nomura, 2011). In higher plants, ppGpp is synthesized in chloroplasts from GTP (GDP) and ATP (Figure 2). Major ppGpp synthase/hydrolase enzymes, named RSH (RelA/SpoT homolog), are localized to chloroplasts (Mizusawa et al., 2008). It has also been reported that ppGpp can negatively regulate chloroplast RNA polymerase (Sato et al., 2009) and the elongation cycle of translation (Nomura et al., 2012). This suggests that ppGpp functions as a regulatory molecule in chloroplasts, and interaction between GKpm and ppGpp may limit the GTP (and ATP) pool, which will subsequently retard chloroplast transcription and translation.

Nucleotide biosynthesis in the cytosol is also involved in the regulation of chloroplast differentiation at early leaf development under cold stress. Yoo et al. (2009) showed that the genes responsible for the $v_{3}$ and st 1 mutants of rice encoded the large and small subunits of ribonucleotide reductase (RNR), RNRL1 and RNRS1, respectively (Yoo et al., 2009). RNR is constructed from large RNR $(\alpha)$ and small RNR $(\beta)$ subunits, which associate to form an active heterodimer complex $\left(\alpha_{2} \beta_{2}\right)$ and catalyze conversion of nucleotide diphosphates (NDPs) to deoxyribonucleotide 
diphosphates (dNDPs; Figure 2). Synthesized dNDPs are rapidly converted into dNTPs for DNA replication and repair. Therefore, the RNR activity affects the entire de novo nucleotide synthesis pathway in vivo (Elledge et al., 1992). As observed in the $v_{3}$ mutant, st1 also caused low-temperature-dependent leaf chlorosis. RNRL1 and RNRS1 abundantly accumulated in the leaves at the P0-P4 stages, and this was enhanced by low temperature (Yoo et al., 2009). Both the $v_{2}$ and $s t 1$ mutations caused missense mutations resulting in reduction of the first ab dimerization, which correlated with the degree of chloroplast disruption (Yoo et al., 2009). This suggests that a threshold level of RNR activity plays an important role in regulating nucleotide flow from the cytosol to chloroplasts. The involvement of cytosolic RNR in plastid nucleotide metabolism is further supported by the report that RNR deficiency causes plastid DNA degradation in pollen in Arabidopsis (Tang et al., 2012). Balancing chloroplast biogenesis and cell division during early leaf development would be achieved through optimization of the nucleotide pool in the cellular compartments.

\section{NUS1 REOUIRED FOR IRNA MATURATION AT LOW TEMPERATURES}

In bacteria, synthesis of ribosomes requires a Rho-dependent antitermination system for the efficient transcription of 16S, 23S, and 5S rRNA from rrn operons (Santangelo and Artsimovitch, 2011). All $r r n$ operons have anti-terminator sequences in their leader and spacer regions, referred to as BoxB, BoxA, and BoxC, that allow RNA polymerase, modified with protein factors, to transcribe rRNA operons. Previously known protein factors that interact with the anti-terminator include NusA, NusB, NusE, and NusG (Santangelo and Artsimovitch, 2011). Recently, Virescent-1 ( $\left.V_{1}\right)$ was identified from a $v_{1}$ mutant of rice and shown to encode a novel chloroplast RNA binding protein, named NUS1 (Kusumi et al., 2011). The C-terminal region of NUS1 has a structural similarity to the RNA-binding domain of the bacterial NusB, which is classified as alpha helical with seven helices. Accumulation of NUS1 specifically occurs in the developing leaves at the P4 stage, and is enhanced by low-temperature treatment (Kusumi et al., 2011). Although there are no regions identical to bacterial Box regions within the chloroplast $r r n$ operon in rice, the gene order of 16S-23S-4.5S-5S and their coding sequences are highly conserved with those of the bacterial $r r n$ operon (Bollenbach etal., 2007). RNA-immunoprecipitation and gel mobility shift assays indicated that NUS1 binds to the upstream leader region of the $16 \mathrm{~S}$ rRNA precursor (Kusumi et al., 2011). The $v_{1}$ mutant had a nonsense mutation in the helical domain and failed to accumulate the NUS1 protein, and therefore probably represents the null phenotype. In the $v_{1}$ seedlings grown at low temperatures, the processing and accumulation of chloroplast rRNA and chloroplast translation/transcription was severely suppressed (Kusumi et al., 1997, 2011). Additionally, Arabidopsis seedlings deficient in a NUS1 ortholog also exhibited a similar phenotype (Kusumi et al., 2011). Therefore, NUS1 is likely to be involved in the regulation of rRNA maturation, which occurs at the P4 stage. Bacterial NusB is involved in the protein complex that interacts with RNA polymerase, nascent mRNA, and ribosomes (Santangelo and Artsimovitch, 2011; Bubunenko et al.,
2013). Recent proteomics-based techniques have allowed the identification of previously uncharacterized proteins that contribute to the chloroplast genetic system (Majeran etal., 2012; Pfalz and Pfannschmidt, 2013). Majeran et al. (2012) showed that in maize, NUS1 and other factors structurally similar to the bacterial Nus-related factors, such as NusG and Rho, were included in nucleoid-enriched fractions. Examination of the physical interactions among these proteins, and identification of other factors interacting with the NUS1 protein, will be vital for elucidating the role of NUS1 in the regulation of the chloroplast genetic system.

\section{CONCLUDING REMARKS}

Compared with other cereals such as wheat and barley, rice is susceptible to cold stress, probably because of its tropical origin (Cruz et al., 2013). The degrees of low-temperature sensitivity and damage vary according to the growth stage. Yoshida (1981) showed that temperature sensitivity varies between stages and that rice plants have a lower threshold temperature for cold damage during the early young seedling stage $\left(10-13^{\circ} \mathrm{C}\right)$ than during the reproductive stage $\left(18-20^{\circ} \mathrm{C}\right)$, making them less sensitive to low temperature as young seedlings. In field conditions, sudden low-temperature phases often occur during the early seedling development in spring. Therefore, it is reasonable to infer that rice developed this mechanism to protect leaf and internal chloroplast development against low temperature-induced retardation.

It has been known that processes of chloroplast translation are sensitive to cold stress. Environmental low temperature arrests protein synthesis by causing ribosomal pausing (Grennan and Ort, 2007) or retardation of ribosomal biogenesis and RNA processing (Millerd et al., 1969; Hopkins and Elfman, 1984; Barkan, 1993). Furthermore, loss of translational factors such as ribosomal protein (Rogalski et al., 2008; Fleischmann et al., 2011; Ehrnthaler et al., 2014; Song et al., 2014), translation elongation factor (Liu et al., 2010), rRNA methylase (Tokuhisa et al., 1998) and the RNA binding protein required for RNA processing (Kupsch et al., 2012) leads to sensitivity to low temperatures. These reports suggest the existence of a particular mechanism that protects chloroplast translation against cold stress, which can be expected to be associated with NUS1, $V_{2}, V_{3}$, and ST1.

The observed involvement of control of translation and nucleotide metabolism in low-temperature tolerance/adaptation has also been reported in bacteria. For example, in Escherichia coli, a temperature downshift hampers ribosome function, and ribosomes change their composition to function properly (Akanuma et al., 2012). It has also been reported that cold-shock proteins are often induced not only by low temperatures but also by translational inhibitors, such as chloramphenicol and tetracycline (Weber and Marahiel, 2003). Therefore, a reduction in translational capacity may be interpreted as a cellular signal triggering the cold adaptation response. Furthermore, analyses of bacterial mutants deficient in ppGpp synthesis showed that artificially induced high levels of ppGpp diminish the expression of cold-shock proteins, while low levels increase their production (Potrykus and Cashel, 2008). ppGpp synthesis is triggered by occupation of the ribosomal A-site by an uncharged tRNA (Potrykus and Cashel, 2008). Considering the hampered ribosomal function at low temperature, 
it is possible that a decrease in cellular ppGpp levels following a temperature downshift plays a physiological role in the regulation of gene expression and adaptation to growth at low temperature. The bacterial NusB protein has also been reported to be involved in cold tolerance. Cells containing a disrupted nusB gene are viable under standard growth conditions, but are cold sensitive (Quan et al., 2005). They are defective in rRNA synthesis and have a decreased peptide elongation rate at low temperatures. NusA, another host factor of the Nus complex, is also induced by cold treatment (Phadtare and Severinov, 2010), suggesting the importance of Nus and the anti-termination system in the cold response in bacteria. These similar properties between the chloroplast and the bacterial low-temperature response imply that higher plants have taken over the bacterial protective system in response to low temperature.

Recently, several other genes have been isolated from low temperature-conditional, chloroplast-deficient mutants of rice, such as OsV4 (virescent 4), wlp1 (white leaf and panicles 1), and tcd9 (thermo-sensitive chloroplast development 9; Gong et al., 2014; Jiang et al., 2014; Song et al., 2014). The corresponding genes in $\mathrm{OsV} 4, w l p 1$, and $t c d 9$ mutants encode plastidial pentatricopeptide repeat (PPR) protein, plastid ribosomal protein L13, and a subunit of chaperonin $60(\mathrm{CP} 60 \alpha)$ required for chloroplast division, respectively. Similarly to NUS1, $V_{2}, V_{3}$, and $S T 1$, their functions are speculated to be involved in early chloroplast development at low temperatures (Gong et al., 2014; Jiang et al., 2014; Song etal., 2014). It is possible that these factors are involved in a closely related mechanism to chloroplast protein expression and assembly, which is required at low temperatures, but not essential for chloroplast development during early leaf development at higher temperatures. Therefore, the maintenance of the developing plastid genetic system will be crucial for tolerance of cold at the seedling stage in rice.

\section{ACKNOWLEDGMENTS}

We are grateful to Dr. Yuzuru Tozawa (Ehime University) for helpful discussion. This work was supported by the Ministry of Education, Culture, Sports, Science, and Technology of Japan (Nos. 21114002, 22570045).

\section{REFERENCES}

Akanuma, G., Nanamiya, H., Natori, Y., Yano, K., Suzuki, S., Omata, S., et al. (2012). Inactivation of ribosomal protein genes in Bacillus subtilis reveals importance of each ribosomal protein for cell proliferation and cell differentiation. J. Bacteriol. 194, 6282-6291. doi: 10.1128/JB.01544-12

Albrecht, V., Ingenfeld, A., and Apel, K. (2006). Characterization of the snowly cotyledon 1 mutant of Arabidopsis thaliana: the impact of chloroplast elongation factor G on chloroplast development and plant vitality. Plant Mol. Biol. 60, 507518. doi: 10.1007/s11103-005-4921-0

Archer, E. K., and Bonnett, H. T. (1987). Characterization of a virescent chloroplast mutant of tobacco. Plant Physiol. 83, 920-925. doi: 10.1104/pp.83.4.920

Asada, K. (1999). The water-water cycle in chloroplasts: scavenging of active oxygens and dissipation of excess photons. Annu. Rev. Plant Physiol. Plant Mol. Biol. 50, 601-639. doi: 10.1146/annurev.arplant.50.1.601

Barkan, A. (1993). Nuclear mutants of maize with defects in chloroplast polysome assembly have altered chloroplast RNA metabolism. Plant Cell 5, 389-402. doi: 10.1105/tpc.5.4.389

Berry, J., and Björkman, O. (1980). Photosynthetic response and adaptation to temperature in higher plants. Annu. Rev. Plant Physiol. 31, 491-543. doi: 10.1146/annurev.pp.31.060180.002423
Bollenbach, T. J., Schuster, G., Portnoy, V., and Stern, D. B. (2007). Processing, degradation and polyadenylation of chloroplast transcripts. Topics Curr. Genet. 19, 175-211. doi: 10.1007/4735_2007_0235

Bubunenko, M., Court, D. L., Al Refaii, A., Saxena, S., Korepanov, A., Friedman, D. I., et al. (2013). Nus transcription elongation factors and RNase III modulate small ribosome subunit biogenesis in Escherichia coli. Mol. Microbiol. 87, 382-393. doi: 10.1111/mmi.12105

Cruz, R. P. D., Sperotto, R. A., Cargnelutti, D., Adamski, J. M., De Freitasterra, T., and Fett, J. P. (2013). Avoiding damage and achieving cold tolerance in rice plants. Food Energy Secur. 2, 96-119. doi: 10.1002/fes3.25

Demmig-Adams, B., and Adams, W. W. (1992). Photoprotection and other responses of plants to high light stress. Annu. Rev. Plant Physiol. Plant Mol. Biol. 43, 599-626. doi: 10.1146/annurev.pp.43.060192.003123

Ehrnthaler, M., Scharff, L. B., Fleischmann, T. T., Hasse, C., Ruf, S., and Bock, R. (2014). Synthetic lethality in the tobacco plastid ribosome and its rescue at elevated growth temperatures. Plant Cell 26, 765-776. doi: 10.1105/tpc.114. 123240

Elledge, S. J., Zhou, Z., and Allen, J. B. (1992). Ribonucleotide reductase: regulation, regulation, regulation. Trends Biochem. Sci. 17, 119-123. doi: 10.1016/09680004(92)90249-9

Fleischmann, T. T., Scharff, L. B., Alkatib, S., Hasdorf, S., Schottler, M. A., and Bock, R. (2011). Nonessential plastid-encoded ribosomal proteins in tobacco: a developmental role for plastid translation and implications for reductive genome evolution. Plant Cell 23, 3137-3155. doi: 10.1105/tpc.111.088906

Gong, X., Su, Q., Lin, D., Jiang, Q., Xu, J., Zhang, J., et al. (2014). The rice OsV4 encoding a novel pentatricopeptide repeat protein is required for chloroplast development during the early leaf stage under cold stress. J. Integr. Plant Biol. 56, 400-410. doi: 10.1111/jipb.12138

Grennan, A. K., and Ort, D. R. (2007). Cool temperatures interfere with D1 synthesis in tomato by causing ribosomal pausing. Photosynth. Res. 94, 375-385. doi: 10.1007/s11120-007-9169-x

Hajdukiewicz, P. T. J., Allison, L. A., and Maliga, P. (1997). The two RNA polymerases encoded by the nuclear and the plastid compartments transcribe distinct groups of genes in tobacco plastids. EMBO J. 16, 4041-4048. doi: 10.1093/emboj/16.13.4041

Hanaoka, M., Kanamaru, K., Fujiwara, M., Takahashi, H., and Tanaka, K. (2005). Glutamyl-tRNA mediates a switch in RNA polymerase use during chloroplast biogenesis. EMBO Rep. 6, 545-550. doi: 10.1038/sj.embor.7400411

Hasanuzzaman, M., Nahar, K., and Fujita, M. (2013). "Extreme temperature responses, oxidative stress and antioxidant defense in plants," in Abiotic Stress - Plant Responses and Applications in Agriculture, eds K. Vahdati and C. Leslie. (Croatia: InTech Open Access Publisher), 169-205. doi: 10.5772/ 54833

Hopkins, W. G., and Elfman, B. (1984). Temperature-induced chloroplast ribosome deficiency in virescent maize. J. Hered. 75, 207-211.

Iba, K., Takamyia, K., Toh, Y., Satoh, H., and Nishimura, M. (1991). Formation of functionally active chloroplast is determined at a limited stage of leaf development in virescent mutants of rice. Dev. Genet. 12, 342-348. doi: 10.1002/dvg.1020120503

Itoh, J., Nonomura, K., Ikeda, K., Yamaki, S., Inukai, Y., Yamagishi, H., et al. (2005). Rice plant development: from zygote to spikelet. Plant Cell Physiol. 46, 23-47. doi: $10.1093 / \mathrm{pcp} / \mathrm{pci501}$

Jarvis, P., and Lopez-Juez, E. (2013). Biogenesis and homeostasis of chloroplasts and other plastids. Nat. Rev. Mol. Cell Biol. 14, 787-802. doi: 10.1038/nrm3702

Jeon, Y., Ahn, C. S., Jung, H. J., Kang, H., Park, G. T., Choi, Y., et al. (2014). DER containing two consecutive GTP-binding domains plays an essential role in chloroplast ribosomal RNA processing and ribosome biogenesis in higher plants. J. Exp. Bot. 65, 117-130. doi: 10.1093/jxb/ert360

Jiang, Q., Mei, J., Gong, X. D., Xu, J. L., Zhang, J. H., Teng, S., et al. (2014). Importance of the rice TCD9 encoding alpha subunit of chaperonin protein 60 (Cpn60 $)$ for the chloroplast development during the early leaf stage. Plant Sci. 215-216, 172-179. doi: 10.1016/j.plantsci.2013.11.003

Kaneda, C., and Beachell, H. M. (1974). Response of indica-japonica rice hybrids to low temperatures. SABRAO J. 6, 17-32.

Kihara, A., Saburi, W., Wakuta, S., Kim, M. H., Hamada, S., Ito, H., et al. (2011). Physiological and biochemical characterization of three nucleoside diphosphate kinase isozymes from rice (Oryza sativa L.). Biosci. Biotechnol. Biochem. 75, 1740-1745. doi: 10.1271/bbb. 110285 
Kupsch, C., Ruwe, H., Gusewski, S., Tillich, M., Small, I., and Schmitz-Linneweber, C. (2012). Arabidopsis chloroplast RNA binding proteins CP31A and CP29A associate with large transcript pools and confer cold stress tolerance by influencing multiple chloroplast RNA processing steps. Plant Cell 24, 4266-4280. doi: 10.1105/tpc.112.103002

Kusumi, K., Chono, Y., Gotoh, E., Tsuyama, M., and Iba, K. (2010a). Chloroplast biogenesis during the early stage of leaf development in rice. Plant Biotechnol. 27 85-90. doi: 10.5511/plantbiotechnology.27.85

Kusumi, K., Hirotsuka, S., Shimada, H., Chono, Y., Matsuda, O., and Iba, K. (2010b). Contribution of chloroplast biogenesis to carbon-nitrogen balance during early leaf development in rice. J. Plant Res. 123, 617-622. doi: 10.1007/s10265-0090277-x

Kusumi, K., Mizutani, A., Nishimura, M., and Iba, K. (1997). A virescent gene $V_{1}$ determines the expression timing of plastid genes for transcription/translation apparatus during early leaf development in rice. Plant J. 12, 1241-1250. doi 10.1046/j.1365-313x.1997.12061241.x

Kusumi, K., Sakata, C., Nakamura, T., Kawasaki, S., Yoshimura, A., and Iba, K. (2011). A plastid protein NUS1 is essential for build-up of the genetic system for early chloroplast development under cold stress conditions. Plant J. 68, 1039 1050. doi: 10.1111/j.1365-313X.2011.04755.x

Liu, X., Rodermel, S. R., and Yu, F. (2010). A var2 leaf variegation suppressor locus, SUPPRESSOR OF VARIEGATION3, encodes a putative chloroplast translation elongation factor that is important for chloroplast development in the cold. BMC Plant Biol. 10:287. doi: 10.1186/1471-2229-10-287

Majeran, W., Friso, G., Asakura, Y., Qu, X., Huang, M., Ponnala, L., et al. (2012) Nucleoid-enriched proteomes in developing plastids and chloroplasts from maize leaves: a new conceptual framework for nucleoid functions. Plant Physiol. 158, 156-189. doi: 10.1104/pp.111.188474

Millerd, A., Goodchild, D. J., and Spencer, D. (1969). Studies on a maize mutant sensitive to low temperature II. Chloroplast structure, development, and physiology. Plant Physiol. 44, 567-583. doi: 10.1104/pp.44.4.567

Mizusawa, K., Masuda, S., and Ohta, H. (2008). Expression profiling of four RelA/SpoT-like proteins, homologues of bacterial stringent factors, in Arabidopsis thaliana. Planta 228, 553-562. doi: 10.1007/s00425-008-0758-5

Nemoto, K., and Yamazaki, K. (1993). "Correlative development of vegetative organs," in Science of the Rice Plant, eds T. Matsuo and K. Hoshikawa (Tokyo: Food and Agriculture Policy Research Center), 625-627.

Nomura, Y., Izumi, A., Fukunaga, Y., Kusumi, K., Iba, K., Watanabe, S., et al. (2014). Diversity in guanosine $3^{\prime}, 5^{\prime}$-bisdiphosphate (ppGpp) sensitivity among guanylate kinases of bacteria and plants. J. Biol. Chem. 289, 15631-15641. doi: 10.1074/jbc.M113.534768

Nomura, Y., Takabayashi, T., Kuroda, H., Yukawa, Y., Sattasuk, K., Akita, M., et al. (2012). ppGpp inhibits peptide elongation cycle of chloroplast translation system in vitro. Plant Mol. Biol. 78, 185-196. doi: 10.1007/s11103-011 9858-x

Omura, T., Satoh, H., Aiga, I., and Nagao, N. (1977). Studies on the character manifestation in chlorophyll mutants of rice: I. Virescent mutants sensitive to low temperature. J. Fac. Agr. Kyushu Univ. 21, 129-140.

Pfalz, J., and Pfannschmidt, T. (2013). Essential nucleoid proteins in early chloroplast development. Trends Plant Sci. 18, 186-194. doi: 10.1016/j.tplants.2012. 11.003

Phadtare, S., and Severinov, K. (2010). RNA remodeling and gene regulation by cold shock proteins. RNA Biol. 7, 788-795. doi: 10.4161/rna.7.6.13482

Potrykus, K., and Cashel, M. (2008). (p)ppGpp: still magical? Annu. Rev. Microbiol. 62, 35-51. doi: 10.1146/annurev.micro.62.081307.162903

Quan, S., Zhang, N., French, S., and Squires, C. L. (2005). Transcriptional polarity in rRNA operons of Escherichia coli nusA and nusB mutant strains. J. Bacteriol. 187, 1632-1638. doi: 10.1128/JB.187.5.1632-1638.2005

Rogalski, M., Schottler, M. A., Thiele, W., Schulze, W. X., and Bock, R. (2008). Rpl33, a nonessential plastid-encoded ribosomal protein in tobacco, is required under cold stress conditions. Plant Cell 20, 2221-2237. doi: 10.1105/tpc.108. 060392

Santangelo, T. J., and Artsimovitch, I. (2011). Termination and antitermination: RNA polymerase runs a stop sign. Nat. Rev. Microbiol. 9, 319-329. doi $10.1038 /$ nrmicro 2560
Sato, M., Takahashi, K., Ochiai, Y., Hosaka, T., Ochi, K., and Nabeta, K. (2009). Bacterial alarmone, guanosine $5^{\prime}$-diphosphate $3^{\prime}$-diphosphate (ppGpp), predominantly binds the $\beta^{\prime}$ subunit of plastid-encoded plastid RNA polymerase in chloroplasts. Chembiochem 10, 1227-1233. doi: 10.1002/cbic.200800737

Song, J., Wei, X., Shao, G., Sheng, Z., Chen, D., Liu, C., et al. (2014). The rice nuclear gene WLP1 encoding a chloroplast ribosome L13 protein is needed for chloroplast development in rice grown under low temperature conditions. Plant Mol. Biol. 84, 301-314. doi: 10.1007/s11103-013-0134-0

Sugimoto, H., Kusumi, K., Noguchi, K., Yano, M., Yoshimura, A., and Iba, K. (2007). The rice nuclear gene, VIRESCENT 2, is essential for chloroplast development and encodes a novel type of guanylate kinase targeted to plastids and mitochondria. Plant J. 52, 512-527. doi: 10.1111/j.1365-313X.2007.03251.x

Sugimoto, H., Kusumi, K., Tozawa, Y., Yazaki, J., Kishimoto, N., Kikuchi, S., et al. (2004). The virescent-2 mutation inhibits translation of plastid transcripts for the plastid genetic system at an early stage of chloroplast differentiation. Plant Cell Physiol. 45, 985-996. doi: 10.1093/pcp/pch111

Suzuki, D. T. (1970). Temperature-sensitive mutations in Drosophila melanogaster. Science 170, 695-706. doi: 10.1126/science.170.3959.695

Tang, L. Y., Matsushima, R., and Sakamoto, W. (2012). Mutations defective in ribonucleotide reductase activity interfere with pollen plastid DNA degradation mediated by DPD1 exonuclease. Plant J. 70, 637-649. doi: 10.1111/j.1365313X.2012.04904.x

Tokuhisa, J. G., Vijayan, P., Feldmann, K. A., and Browse, J. A. (1998). Chloroplast development at low temperatures requires a homolog of DIM1, a yeast gene encoding the $18 \mathrm{~S}$ rRNA dimethylase. Plant Cell 10, 699-711. doi: 10.1105/tpc.10.5.699

Tozawa, Y., and Nomura, Y. (2011). Signalling by the global regulatory molecule ppGpp in bacteria and chloroplasts of land plants. Plant Biol. 13, 699-709. doi: 10.1111/j.1438-8677.2011.00484.x

Verstraeten, N., Fauvart, M., Versees, W., and Michiels, J. (2011). The universally conserved prokaryotic GTPases. Microbiol. Mol. Biol. Rev. 75, 507-542. doi: 10.1128/MMBR.00009-11

Weber, M. H., and Marahiel, M. A. (2003). Bacterial cold shock responses. Sci. Prog. 86, 9-75. doi: 10.3184/003685003783238707

Yagi, Y., and Shiina, T. (2014). Recent advances in the study of chloroplast gene expression and its evolution. Front. Plant Sci. 5:61. doi: 10.3389/fpls.2014.00061

Yamori, W., Sakata, N., Suzuki, Y., Shikanai, T., and Makino, A. (2011). Cyclic electron flow around photosystem I via chloroplast $\mathrm{NAD}(\mathrm{P}) \mathrm{H}$ dehydrogenase (NDH) complex performs a significant physiological role during photosynthesis and plant growth at low temperature in rice. Plant J. 68, 966-976. doi: 10.1111/j.1365-313X.2011.04747.x

Yoo, S.-C., Cho, S.-H., Sugimoto, H., Li, J., Kusumi, K., Koh, H.-J., et al. (2009). Rice Virescent- 3 and Stripe- 1 encoding the large and small subunits of ribonucleotide reductase are required for chloroplast biogenesis during early leaf development. Plant Physiol. 150, 388-401. doi: 10.1104/pp.109.136648

Yoshida, S. (1981). "Growth and development of the rice plant," in Fundamentals of Rice Crop Science. (Los Baños: Philippines: International Rice Research Institute), $1-63$.

Conflict of Interest Statement: The authors declare that the research was conducted in the absence of any commercial or financial relationships that could be construed as a potential conflict of interest.

Received: 17 May 2014; accepted: 20 July 2014; published online: 11 August 2014. Citation: Kusumi K and Iba K (2014) Establishment of the chloroplast genetic system in rice during early leaf development and at low temperatures. Front. Plant Sci. 5:386. doi: $10.3389 / f p l s .2014 .00386$

This article was submitted to Plant Physiology, a section of the Journal Frontiers in Plant Science.

Copyright $(2014$ Kusumi and Iba. This is an open-access article distributed under the terms of the Creative Commons Attribution License (CC BY). The use, distribution or reproduction in other forums is permitted, provided the original author (s) or licensor are credited and that the original publication in this journal is cited, in accordance with accepted academic practice. No use, distribution or reproduction is permitted which does not comply with these terms. 